Editorial Perspective

\title{
The hyperfunction theory of aging: three common misconceptions
}

\author{
Mikhail V. Blagosklonny ${ }^{1}$ \\ ${ }^{1}$ Roswell Park Cancer Institute, Buffalo, NY 14263, USA \\ Correspondence to: Mikhail V. Blagosklonny, email: Blagosklonny@oncotarget.com, Blagosklonny@rapalogs.com \\ Keywords: geroscience; rapamycin; mTOR; geroconversion; quasi-program; cell senescence
}

Received: September 08, $2021 \quad$ Accepted: September 14, $2021 \quad$ Published: September 17, 2021

Copyright: ( 2021 Blagosklonny. This is an open access article distributed under the terms of the Creative Commons Attribution License (CC BY 3.0), which permits unrestricted use, distribution, and reproduction in any medium, provided the original author and source are credited.

Almost all papers on aging start with the statement that aging is functional decline caused by accumulation of molecular damage. In contrast, according to the hyperfunction theory, aging is not functional decline, but results from cellular hyperfunctions that produce agerelated diseases. The sum of these age-related diseases is aging (Figure 1). Functional decline is secondary to primary hyperfunctions. Second, aging is not caused by the accumulation of molecular damage - it is caused by inappropriate activation of signaling pathways, such as mTOR. These signaling pathways directly drive agerelated diseases. The hyperfunction theory of aging was discussed in detail in numerous papers (just to name a few [1-10]). However, given the unconventional nature of the hyperfunction theory, some points are commonly misunderstood. Here, I will address three common misconceptions people tend to have about the hyperfunction theory, without discussing the theory in detail.

\section{MISCONCEPTION 1}

Hyperfunction is always an increase of function. Correctly, hyperfunction is often an unchanged function, that is still higher than optimal for longevity

Hyperfunction is a function that was not switched off upon its completion [1]. In some cases, age-related alterations are indeed an absolute increase: hypersecretory phenotype, pro-inflammation, hypertension, hyperlipidemia, hyperglycemia, hyperinsulinemia, hyperplasia and hypertrophy of cells and organs (e.g., heart and prostate). In typical cases, hyperfunction is relative. It may even be a decrease of function that is still higher than optimal for longevity in the aging organism (Figure 2).

Using an analogy, consider a car driving 65 miles per hour (mph) on the highway with a $65 \mathrm{mph}$ speed limit. This is the normal and optimal speed on this highway, or optimal functioning early in life. Early in life, during

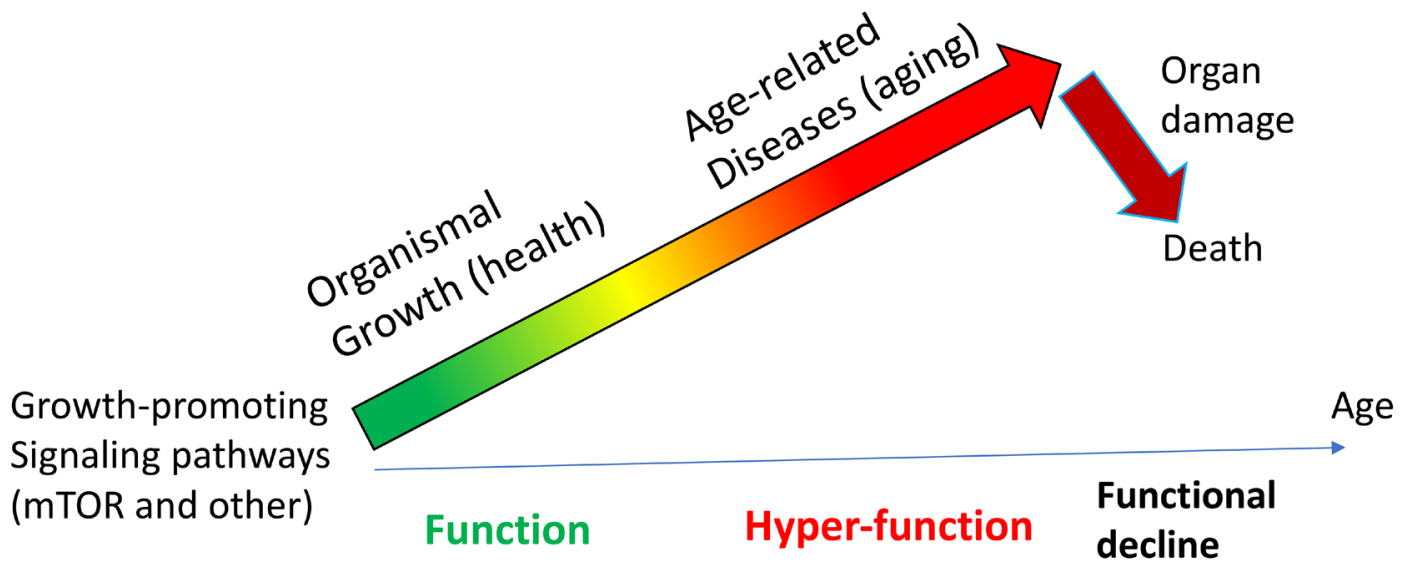

Figure 1: General presentation of hyperfunction theory. Aging is a hyper-function caused by unnecessary and persistently activated signaling pathways, such as mTOR (for example), not by molecular damage. These signaling pathways activate cells, directly causing the development of age-related diseases. Aging is a sum of all diseases. Hyperfunctions may eventually lead to organ damage and loss of functions. Adopted with modifications from Figure 1 in ref. [1]. 
organism growth, all cellular and systemic functions are optimal for growth (not for longevity). However, if the car exits the highway to enter low-speed streets without decreasing speed (stuck accelerator) and continues at the same speed, $65 \mathrm{mph}$ becomes over-speeding, or hyperfunction. The car is bound to crash on your driveway and is destroyed by over-speeding. It has no chance to be destroyed on a molecular level by rusting. Similarly, hyperfunction causes organ damage (e.g. stroke, infarction, cancer metastasis, broken hip) and death before molecular damage (rusting by free radicals) accumulates to deadly levels [2].

\section{MISCONCEPTION 2}

\section{Hyperfunction vs. molecular damage}

The second misconception is that the hyperfunction theory of aging denies a harmful accumulation of molecular damage. To clarify, molecular damage does accumulate. Furthermore, molecular damage would eventually kill the organism, unless the organism dies from hyperfunctional aging or, even more specifically, from mTOR-driven aging (Figure 3). Aging due to molecular damage and due to cellular hyperfunctions

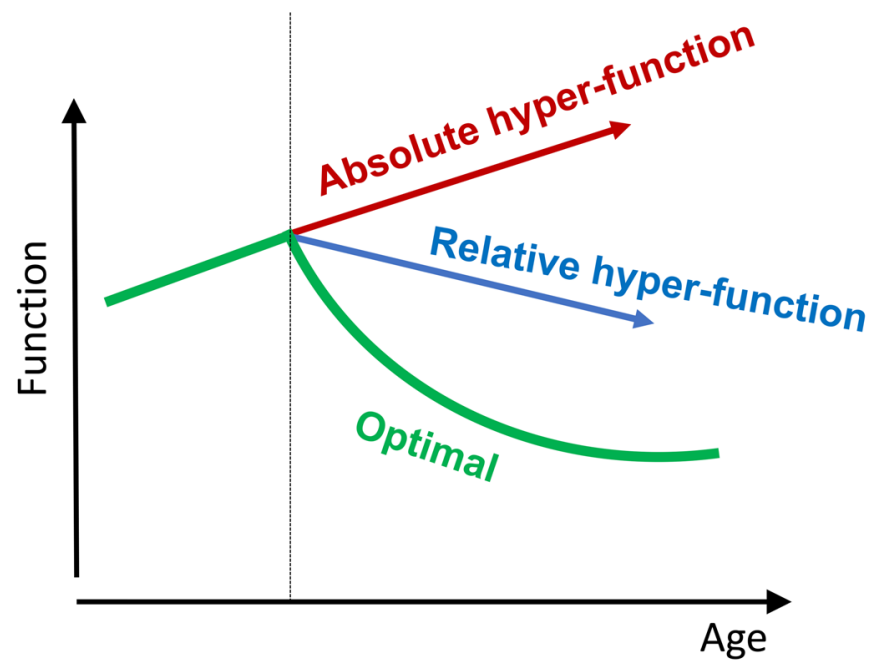

Figure 2: Absolute and relative hyperfunctions. Optimal function is age-dependent.
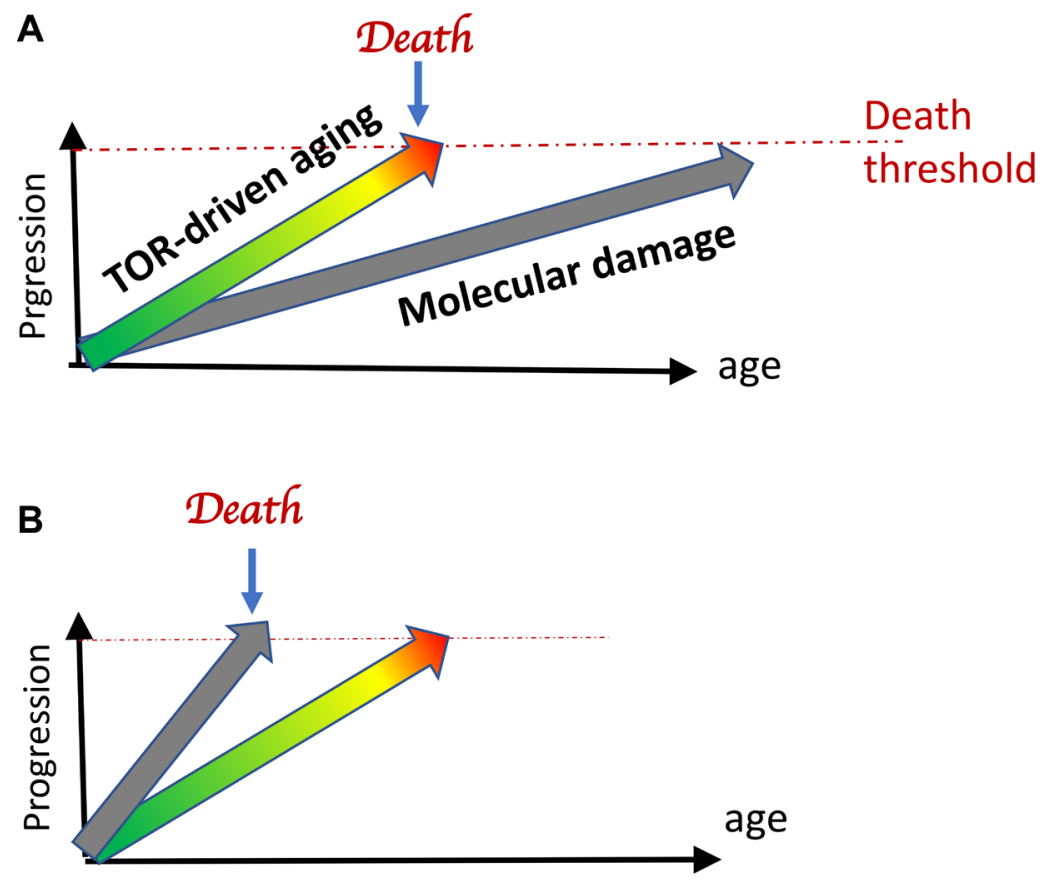

Figure 3: Life-limiting aging. (A) Normal Aging. Hyperfunctional (mTOR-driven) aging is life-limiting. It reaches a deadly threshold earlier than accumulating molecular damage does. (B) Premature aging syndromes. When artificially accelerated by gene knockouts, accumulation of molecular damage may become life-limiting. Adopted from ref. [10]). 
occur in parallel, but the latter is a life-limiting process, which progresses faster.

How do we know that hyperfunctional aging is lifelimiting and accumulation of molecular damage is not? In several dozen studies, rapamycin (mTORC1 inhibitor) prolonged lifespan in animals (see for references $[10,11]$ ). In some short-lived mice, rapamycin even tripled maximal lifespan [12]. Then mTOR-driven aging is life-limiting almost by definition.

In contrast, a decrease of molecular damage does not prolong lifespan in most studies. Interestingly, a very mild increase of molecular damage may prolong lifespan, probably by inhibiting mTOR [13]. To make molecular damage life-limiting, it is necessary to dramatically increase it by knocking out key enzymes, telomerases, and so on [10]. Of note, symptoms of molecular damage-induced premature aging are different from normal age-related diseases. In addition, hyperfunctional aging must be lifelimiting from a theoretical perspective, and it was predicted in 2006 (before rapamycin was tested in animals) that rapamycin must extend lifespan in animals and humans [1].

\section{MISCONCEPTION 3}

The hyperfunction theory is primarily based on an evolutionary theory. Correctly, the hyperfunction theory is principally based on a cellular model of geroconversion

The hyperfunction theory is not just an evolutionary theory, even though it is completely in agreement with the latter and develops the notion of Antagonistic Pleiotropy (AP) further. Evolutionary perspectives in the hyperfunction theory are needed mostly to explain why hyperfunctional (quasi-programmed) aging is life-limiting and why accumulation of molecular damage is not [9]. (Note: Hyperfunction/quasi-program is an aimless and harmful continuation of a program that was not switched off upon its completion.)

Otherwise, the hyperfunction theory is a mechanistic theory: an analogy of the cellular model of geroconversion in vitro (Figure 4 ). When cells proliferate, mTOR and other growth-promoting signaling pathways drive cellular mass growth, which is balanced by cell division. However, if the cell cycle is blocked by p21 or p16, then the same mTOR pathway drives "pathological growth" (geroconversion) from reversible arrest to irreversible senescence [14]. Geroconversion is a continuation of growth - a quasi-program of growth. The arrested cells grow in size exponentially [15] (without division) and become large, flat, and hyperfunctional, namely beta Galpositive (lysosomal hyperfunction), SASP (hyper-secretory phenotype), and compensatory insulin/growth factorresistant [16, 17], as could be predicted on theoretical grounds [18]. The hyperfunction theory was derived from cancer research because the same signaling pathways are involved in both cancer and geroconversion.

The hyperfunction theory is a translation of the rules of geroconversion to the organism. Organismal aging and geroconversion can be described in similar terms, and similar signaling pathways drive geroconversion and organismal aging [1].
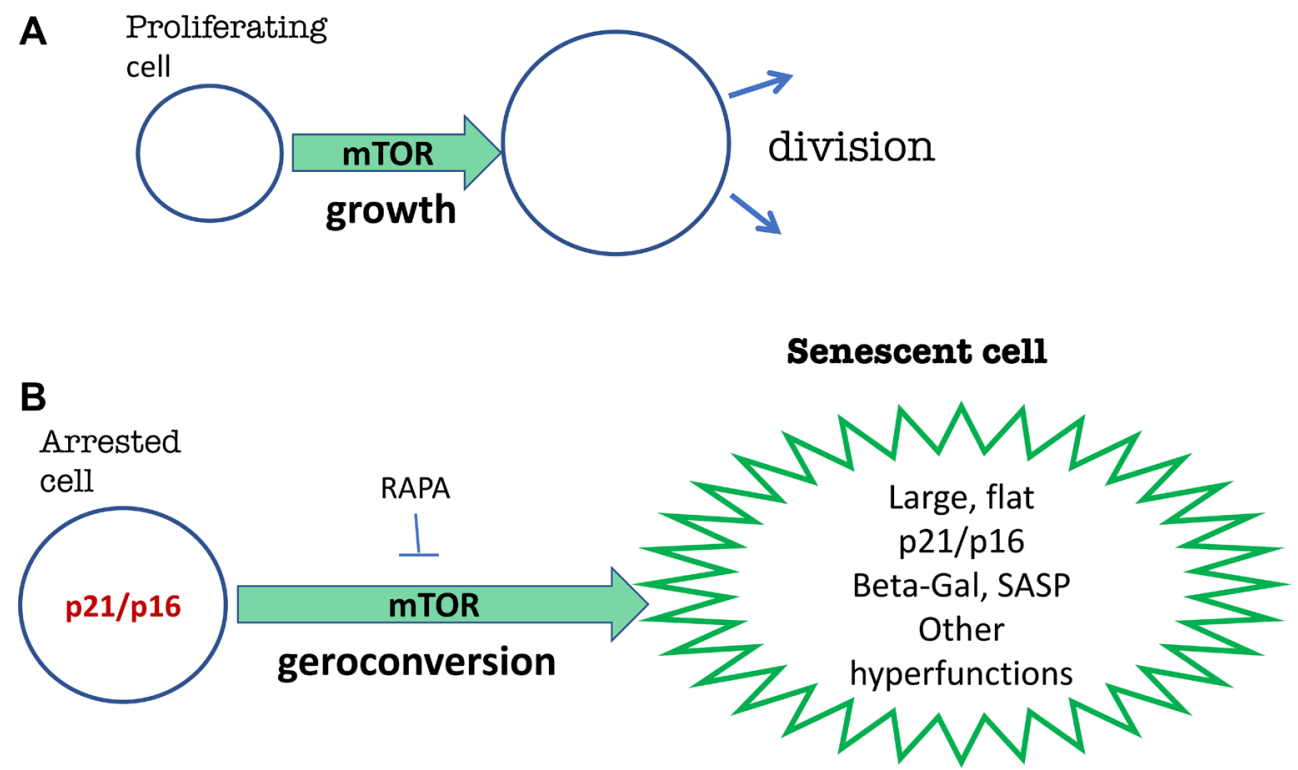

Figure 4: Geroconversion model (in cell culture) displays basic features of hyperfunction theory. (A) In proliferating cells, growth-promoting pathways such as mTOR drive cell mass growth, which is balanced by cell division. (B) When the cell cycle is suddenly blocked by $\mathrm{p} 21$ and p16, growth-promoting pathways such as mTOR drive geroconversion to senescence. Proliferation-like activity of mTOR in non-proliferating cells is a hyperfunction. Senescent cells display various hyperfunctions on a cellular level. See text for details. 
It does not necessarily mean that a few senescent cells cause organismal aging. Fully senescent cells may contribute to aging, but are not required [19]. Instead, most cells are becoming at least relatively hyperfunctional, gerogenic, producing age-related diseases, as exemplified by the development of atherosclerosis and hypertension, which may culminate in infarction and death [20]. The geroconversion model is a simplified model of quasiprogrammed or hyperfunctional organismal aging.

On an organismal level, the hyperfunction theory describes the development of age-related diseases [20]. David Gems and co-workers demonstrated that age-related diseases in C. elegans are quasi-programmed, developing a fruitful model for the hyperfunction theory [8, 21-23]. Even aging in yeast remotely models the hyperfunction theory, and mTOR was identified as one of drivers of yeast aging $[24,25]$.

Geroconversion is driven by continuously active growth-promoting signaling pathways such as PI3K/ mTOR and MEK/MAPK, which are maximally activated in proliferating cells and continue to be active through external signaling (e.g., insulin, nutrients, GFs), which establishes positive feedback loops in the organism. Geroconversion is not driven by the genome, although gene expression changes dramatically during geroconversion, partially ensuring its irreversibility.

The idea that aging is a continuation of development is not new [26-28] and, as a general notion, was entertained even before the term gerontology was coined [28]. As put by Walker, who revisited developmental theories, "developmental inertia is the primary cause of senescence". [29] Without specific mechanisms, the idea is too general or vague to have clinical applications. The developmental theory of aging by Dilman implicated one organ - the hypothalamus (a tiny part of the brain) [26, 27]. I discussed this developmental theory of aging in the light of the hyperfunction theory [30].

The essence of hyperfunction theory is that, later in life, higher than optimal activity of signaltransduction pathways (maintained by positive feedback loops) directly drives age-related diseases (which are, in sum, aging). This notion is most appealing to practicing physicians, who have implemented this theory in the treatment of age-related diseases by slowing aging https:// rapamycintherapy.com/.

In sum, the hyperfunction theory of organismal aging was initially developed as an analogy to the geroconversion model. The geroconversion model makes it possible to discover anti-aging drugs (Figure 4), because drugs that inhibit geroconversion slow down organismal aging. Rapamycin slows geroconversion, predicting that rapamycin would increase the lifespan of animals. Therefore, in 2006, it was suggested that rapamycin (clinically available since 1999) can be used in humans immediately to slow down aging and all age-related diseases [1].

\section{CONFLICTS OF INTEREST}

Authors have no conflicts of interest to declare.

\section{REFERENCES}

1. Blagosklonny MV. Aging and immortality: quasiprogrammed senescence and its pharmacologic inhibition. Cell Cycle. 2006; 5:2087-102. https://doi.org/10.4161/ cc.5.18.3288. PMID: 17012837

2. Blagosklonny MV. TOR-driven aging: speeding car without brakes. Cell Cycle. 2009; 8:4055-59. https://doi. org/10.4161/cc.8.24.10310. PMID:19923900

3. Blagosklonny MV. Rapamycin and quasi-programmed aging: four years later. Cell Cycle. 2010; 9:1859-62. https:// doi.org/10.4161/cc.9.10.11872. PMID:20436272

4. Blagosklonny MV. Answering the ultimate question "what is the proximal cause of aging?". Aging (Albany NY). 2012; 4:861-77. https://doi.org/10.18632/aging.100525. PMID:23425777

5. Gems D, de la Guardia Y. Alternative Perspectives on Aging in Caenorhabditis elegans: Reactive Oxygen Species or Hyperfunction? Antioxid Redox Signal. 2013; 19:321-29. https://doi.org/10.1089/ars.2012.4840. PMID:22870907

6. Gems D, Partridge L. Genetics of longevity in model organisms: debates and paradigm shifts. Annu Rev Physiol. 2013; 75:621-44. https://doi.org/10.1146/annurevphysiol-030212-183712. PMID:23190075

7. Gems D. The Hyperfunction Theory: An Emerging Paradigm for the Biology of Aging. Preprints. 2021; 2021080552. https://doi.org/10.20944/preprints202108.0552.v1.

8. Wang H, Zhao Y, Ezcurra M, Benedetto A, Gilliat AF, Hellberg J, Ren Z, Galimov ER, Athigapanich T, Girstmair J, Telford MJ, Dolphin CT, Zhang Z, Gems D. A parthenogenetic quasi-program causes teratoma-like tumors during aging in wild-type $C$. elegans. NPJ Aging Mech Dis. 2018; 4:6. https://doi.org/10.1038/s41514-018-0025-3. PMID:29928508

9. Blagosklonny MV. Response to the Thought-Provoking Critique of Hyperfunction Theory by Aubrey de Grey. Rejuvenation Res. 2021; 24:170-72. https://doi. org/10.1089/rej.2021.0018. PMID:33784825

10. Blagosklonny MV. DNA- and telomere-damage does not limit lifespan: evidence from rapamycin. Aging (Albany NY). 2021; 13:3167-75. https://doi.org/10.18632/ aging.202674. PMID:33578394

11. Swindell WR. Rapamycin in mice. Aging (Albany NY). 2017; 9:1941-42. https://doi.org/10.18632/aging.101289. PMID:28876223

12. Johnson SC, Yanos ME, Kayser EB, Quintana A, Sangesland M, Castanza A, Uhde L, Hui J, Wall VZ, Gagnidze A, Oh K, Wasko BM, Ramos FJ, et al. mTOR inhibition alleviates mitochondrial disease in a mouse 
model of Leigh syndrome. Science. 2013; 342:1524-28. https://doi.org/10.1126/science.1244360. PMID:24231806

13. Blagosklonny MV. Hormesis does not make sense except in the light of TOR-driven aging. Aging (Albany NY). 2011; 3:1051-62. https://doi.org/10.18632/aging.100411. PMID:22166724

14. Demidenko ZN, Blagosklonny MV. Growth stimulation leads to cellular senescence when the cell cycle is blocked. Cell Cycle. 2008; 7:3355-61. https://doi.org/10.4161/ cc.7.21.6919. PMID:18948731

15. Demidenko ZN, Blagosklonny MV. Quantifying pharmacologic suppression of cellular senescence: prevention of cellular hypertrophy versus preservation of proliferative potential. Aging (Albany NY). 2009; 1:100816. https://doi.org/10.18632/aging.100115. PMID:20157583

16. Blagosklonny MV. Rapamycin, proliferation and geroconversion to senescence. Cell Cycle. 2018; 17:265565. https://doi.org/10.1080/15384101.2018.1554781. PMID:30541374

17. Velarde MC, Demaria M, Campisi J. Senescent cells and their secretory phenotype as targets for cancer therapy. Interdiscip Top Gerontol. 2013; 38:17-27. https://doi. org/10.1159/000343572. PMID:23503512

18. Blagosklonny MV. Cell senescence and hypermitogenic arrest. EMBO Rep. 2003; 4:358-62. https://doi.org/10.1038/ sj.embor.embor806. PMID:12671679

19. Blagosklonny MV. Anti-aging: senolytics or gerostatics (unconventional view). Oncotarget. 2021; 12:1821-35. https://doi.org/10.18632/oncotarget.28049. PMID:34504654

20. Blagosklonny MV. Prospective treatment of age-related diseases by slowing down aging. Am J Pathol. 2012; 181:1142-46. https://doi.org/10.1016/j.ajpath.2012.06.024. PMID:22841821

21. de la Guardia Y, Gilliat AF, Hellberg J, Rennert P, Cabreiro $\mathrm{F}$, Gems D. Run-on of germline apoptosis promotes gonad senescence in C. elegans. Oncotarget. 2016; 7:39082-96. https://doi.org/10.18632/oncotarget.9681. PMID:27256978

22. Ezcurra M, Benedetto A, Sornda T, Gilliat AF, Au C, Zhang Q, van Schelt S, Petrache AL, Wang H, de la Guardia Y, Bar-Nun S, Tyler E, Wakelam MJ, Gems D. C. elegans Eats Its Own Intestine to Make Yolk Leading to Multiple Senescent Pathologies. Curr Biol. 2018; 28:2544-56.e5. https://doi.org/10.1016/j.cub.2018.06.035. PMID:30100339
23. Wang H, Zhang Z, Gems D. Monsters in the uterus: teratoma-like tumors in senescent $C$. elegans result from a parthenogenetic quasi-program. Aging (Albany NY). 2018; 10:1188-89. https://doi.org/10.18632/aging.101486. PMID:29923830

24. Powers RW 3rd, Kaeberlein M, Caldwell SD, Kennedy BK, Fields S. Extension of chronological life span in yeast by decreased TOR pathway signaling. Genes Dev. 2006; 20:174-84. https://doi.org/10.1101/gad.1381406. PMID: 16418483

25. Kaeberlein M, Powers RW 3rd, Steffen KK, Westman EA, Hu D, Dang N, Kerr EO, Kirkland KT, Fields S, Kennedy BK. Regulation of yeast replicative life span by TOR and Sch9 in response to nutrients. Science. 2005; 310:1193-96. https://doi.org/10.1126/science.1115535. PMID:16293764

26. Dilman VM. Age-associated elevation of hypothalamic, threshold to feedback control, and its role in development, ageine, and disease. Lancet. 1971; 1:1211-19. https://doi. org/10.1016/s0140-6736(71)91721-1. PMID:4103080

27. Dil'man VM. Transformation of the development program in the mechanism of age pathology. An elevational model of age pathology and natural death in man. Hum Physiol. 1978; 4:465-78. PMID:381162

28. Agronin ME. From Cicero to Cohen: developmental theories of aging, from antiquity to the present. Gerontologist. 2014; 54:30-39. https://doi.org/10.1093/ geront/gnt032. PMID:23665459

29. Walker RF. Developmental theory of aging revisited: focus on causal and mechanistic links between development and senescence. Rejuvenation Res. 2011; 14:429-36. https://doi. org/10.1089/rej.2011.1162. PMID:21767161

30. Blagosklonny MV. M(o)TOR of aging: MTOR as a universal molecular hypothalamus. Aging (Albany NY). 2013; 5:490-94. https://doi.org/10.18632/aging.100580. PMID:23872658 